"Why am I short of breath, doctor?"

SIR,-In the images in the cardiology section (page 312) of the October 1994 issue Dr Davies presented an unusual case of poor cardiac output. ${ }^{1}$ The discussion of this case raises a perennial issue - that of the cause of dyspnoea in heart diseases, including heart failure.

Dr Davies stated that in his patient the dyspnoea on exertion was caused by poor cardiac output that was the result of inadequate venous return. This improved when the dose of diuretic was reduced. This seems a classic case of, to use the old-fashioned term, forward cardiac failure rather than the backward cardiac failure, which would present with pulmonary congestion. Though Dr Davies' observations are undoubtedly correct, they raise yet again the question why this man was dyspnoeic when there is no description of any pulmonary congestion, and when, in fact, the implication was that the patient was somewhat hypovolaemic. These patients complain of dyspnoea well before the onset of lactic acidosis, so that this alone is not a sufficient explanation.

For several years we have been studying the mechanisms underlying the excessive ventilatory response to exercise in patients with chronic heart failure in whom the dose of diuretic is adequate. We have evidence that one contributory factor may be an abnormally active muscle signal transmitted via unmyelinated afferents from so called "ergoreceptors" within skeletal muscle. This explanation has the advantage of combining the mechanisms of fatigue and dyspnoea in one mechanism - that of abnormal peripheral skeletal muscle, which then sends an abnormally active signal to the cortex, mediating both fatigue and dyspnoea. Both end stage liver disease and some of the nonmetastatic manifestations of neoplastic disease can present with unexplained fatigue and dyspnoea. Both conditions are also frequently associated with a peripheral myopathy.

Throughout these studies I have been surprised, even amazed, by the strength of the firmly held belief that we already know the cause of dyspnoea in heart failure. Even to question whether pulmonary congestion is the primary cause is met with incredulity, and if expert referees' reports on grant applications are the yardstick, then clearly even expert opinion does not accept there is a problem to investigate. Yet despite this we see case after case in which the accepted explanations for the generation of dyspnoea are patently unable to explain this disabling symptom.

Could this be another case of the facts getting in the way of firmly held belief? ANDREW J COATS Royal Brompton National Heart and Lung Institute, Dovehouse Street,
London SW3 $6 L Y$

1 Davies MK. An unusual cause of poor cardiac output. Br Hearn $\mathcal{F} 1994 ; 72: 312$.

\section{Anticoagulation after intracoronary stent insertion}

SIR,-Anticoagulation after intracoronary stent insertion is a controversial issue and, because the anticoagulation protocols used in different hospitals are short-lived, nurses and junior staff have to adjust to everchanging protocols at a rapid pace. The article by Brack et al gives the impression that the proposed anticoagulation strategy is an accepted mode of treating patients after stent implantation. ${ }^{1}$ This is misleading and requires comment.

The paper was accepted for publication on the 9 February 1994. In the meantime most institutions have relaxed their anticoagulation scheme considerably. As Brack $e$ al state in their last sentence, low molecular weight heparin has replaced intravenous heparin in many centres and no anticoagulation variables are monitored. The relatively elaborate protocol proposed in the article adds considerably to the work of the nursing staff and to the cost. Also, heavy anticoagulation can cause several local and systemic complications. ${ }^{2}$ The fact that Brack et al saw no stent thrombosis in their last 50 procedures after this relatively strict anticoagulation protocol does not mean that the antithrombotic regimen was responsible for the good outcome. It may well be that the learning curve of the stent implantation overlaid use of this protocol. Most investigators involved in this topic now agree that it is the primary result after stenting that determines the probability of thrombotic occlusion rather than the anticoagulation regimen. If the primary result is impeccable without any residual stenosis (as judged by digital angiography or intravascular ultrasound) and with excellent apposition of all stent struts in all segments and good flow, anticoagulation with heparin and warfarin can probably be dispensed with. ${ }^{3}$ Aspirin may then be the only recommended treatment.

I recommend that heavy anticoagulation be reserved for cases where the results of stenting are less than perfect.

ULRICH SIGWART Department of Invasive Cardiology Royal Brompton Hospital, Sydney Street,

1 Brack MJ, Hubner PJB, London SW3 6NP Anticoagulation after intracoronary stent insertion. Br Heart $\mathcal{F} 1994 ; 72: 294-6$.

2 Brown DL, MacIsaac AI, Topol EJ. Pulmonary hemorrhage after intracoronary stent placement. $\Im \mathrm{Am}$ Coll Cardiol 1994;24:91-4.

3 Colombo A, Hall P, Almagor Y, Maiello L, Gaglione A, Nakamura S, et al. Results of intravascular ultrasound guided coronary intravascular ultrasound guided coronary [abstr]. $\Im$ Am Coll Cardiol 1994;23:335.

This letter was shown to the authors, who reply as follows:

SIR-We read Dr Sigwart's letter with interest and agree with some of the points he is making. Clearly with a technique that is changing as fast as intracoronary stenting any review article will lose currency in the months between acceptance and publication.

There is no doubt that (sub)acute closure after stenting is less common when the stent is placed de novo than when the stent is placed as a bail out procedure. The mean closure rates that are quoted range from $3-4 \%$ for de novo stenting to $14-20 \%$ for bail out. Thus de novo stenting will carry with it a smallish risk of stent closure and as Dr Sigwart has indicated the incidence will relate to the angiographic appearance. We are not certain how an "impeccable" result compares with a very good result and whether any difference will have an impact on clinical outcome. Additionally, whether the differences can be judged angiographically will depend on the quality of the radiological image. When the angiographic result is very good the extra value of information provided by intravascular ultrasound (IVU) is also uncertain. Most operators rely on angiographic rather than IVU evaluation of stent placement.

In the Benestent I trial (sub)acute closure was $3.5 \%$, confirming that closure is not common for de novo stenting. Most operators in the U.K, however, are stenting because of a poor angiographic result or to bail out after dissection or vessel closure. Such an environment is very different from that in de novo stenting and potentially is very prothrombotic (release of tissue factor, activation of the coagulation cascade, reduction in flow). We suggest that any messages from de novo stenting (as per Benestent I and subsequently Benestent II) may not be applicable to unplanned stenting.

We agree with Dr Sigwart that the peripheral side effects of anticoagulation should not be underestimated. It is for this reason we continue to believe that if anticoagulation is to be undertaken rigid monitoring and leaving sheaths in position until the activated partial thromboplastin time has fallen will lessen the risk of peripheral complications.

Thus while we accept Dr Sigwart's comments about the change in clinical experience, which suggest that there may no longer be a need for such rigid anticoagulation in de novo stenting, any stenting indication that differs from this will also need to be tested. In the meantime to ensure appropriate well controlled anticoagulation we will continue to adhere to our protocol for unplanned stenting.

Our article set out to suggest that anticoagulation if it is to be undertaken should be done properly. Rather than suggest that our outline was an accepted protocol, as $\mathrm{Dr}$ Sigwart suggests in his first paragraph, we are proposing that it should be.

$M$ BRACK P J B HUBNER A H GERSHLICK Department of Cardiology, Glenfield General Hospital, Groby Road, Leicester Cardiac Society will take place at the Conference Centre, Harrogate, North Yorkshire from 23 to 25 May.

A course on practical adult cardiovascular pathology organised by Professor M J Davies and Dr M N Sheppard will take place at the Royal Brompton National Heart and Lung Institute on Monday 16 October 1995. For further information, please contact Dr Mary N Sheppard, National Heart and Lung Institute, Dovehouse Street, London SW3 6LY (tel: 0171351 8172; fax: 01713763442 ).

The ninth annual meeting of the Mediterranean Association of Cardiology and Cardiac Surgery will take place on 20-25 October 1996 in Tel Aviv, Israel. For further information, please contact The Secretariat, 9th Annual Meeting of Cardiology and Cardiac Surgery, PO Box 50006, Tel Aviv 61500, Israel (tel: +972 3514 0014; fax: +972 3 517 5674/514 0077). 